\title{
Melanosis lencutilaris progressiva (Pick), Xeroderma pigmentosum (Kaposi).
}

\author{
Von \\ Dr. Edmund Stern, \\ Specialarzt für Hautkrankheiten in Mannheim.
}

(Hiezu Tafel XII.)

Den etwa 53 bisher beschriebenen ') Fällen dieser merkwürdigen Hauterkrankung möchte ich in Folgendem einen weiteren kurz hinzufügen. Es is t d e r erste aus Südde ut s chland und ein neuer Beleg für die allerdings nur von NichtSpecialisten bestrittene Ubiquität der Hautrankheiten.

Johannes P., 12 Jahre alt, Sohn des Bahnarbeiters Jakob P. zn H. Aufgen. 8. Juli 1888.

An a mn es e: Belkam im 6. Lebensmonate einen ,grindigen, nässenden Ausschlag" besonders der linken Gesichtshälfte, der von selbst heilte. Als nun der Knabe an einem Darmcatarrh erkrankte, sagte der Arzt, der Ausschlag sei zurückgetrieben und ricf ihn durch äussere Mittel (wodurch, ist nicht genau eruirbar, wahrscheinlich Schmierseife) wieder hervor, worauf derselbe noch ein Jahr bestand und dann heilte. Gegen das Ende des 2. Lebensjahres bedeckte sich das ganze Gesicht mit zahlreichen Sommersprossen, obwohl der Knabe infolge seiner. Kränkljehkeit nur wenig ins Freie gebracht wurde. Im 8. Jahre überstand er eine Lungrenentzündung.

Die jetzige Krankheit begann vor 2 Jahren mit einem rothen Knötchen an der linken Schläfe, das 6 Wochen nach seinem Erscheinen aufbrach und von einem Bader mit Höllenstein geätzt wurde. Der später zuge-

1) Elsenberg hat in seiner Zusammenstellung (dieses Archiv 1890. p. 50) den Fall von Köbner (Block, dreitausend Fälle. Berlin 1888. p. 54) übersehen. 
zogene Arzt verordnete eine Salbe durch 2 Monate und schickte dann, da keine Besserung eintrat, den Knaben in eine chirurgische Klinik, wo die kranke Stelle herausgeschnitten wurde. Spitalsaufenthalt 3 Monate. Der Kranke wurde mit noch nicht geschlossener Wunde entlassen, die langsam zuheilte, aber nach 2 Monaten wieder aufbrach. Zugleich bildeten sich vor dem linken Obre Anschwellungen. Da sich auch Anfälle von heftigem Kopfschmerz und Erbrechen einstellten, so wandten sich die Eltern an mich.

Familie. In der Familie des Vaters sind rothe Haare und Sommersprossen auf Gesicht und Vorderarmen heimisch. Von den 5 Geschwistern unseres Kranken, deren ältestes er ist, haben alle rothe Haare und auffallend helle Hautfarbe, drei weisen Sommersprossen, eines ein 11 jähriges Mädchen, neben zahlreichen Epheliden im Gesicht und auf den Vorderarmen, auch Teleangiektasien daselbst auf. Beide Eltern sind völlig gesund und Hautkrankheiten sind in der Familie noch nicht vorgekommen.

Stat. praes. (8. Juli 1888.) Für sein Alter mässig entwickelter, magerer, leidend aussehender Knabe. Haut im Allgemeinen normal, aber sehr hlass, fast weiss. Haare braungelb, glatt. Iris grün. Zähne mit Ausnahme einiger variöser Backzähne intact. Auge und Ohr sowie die inneren Organe gesund. Erkrankt ist nur Gesicht und Hals. Stirn, Schläfen, Nase, Wangen, Kinn und Ohren sind mit dicht stehenden punkt- und streifenförmigen, rundlichen und sternartigen hellbräunlichen, auf Druck nicht erblassenden Flecken bedeckt, zwischen denen sich spärliche, aber ziemlich grosse Teleangiektasien vorfinden. Diese als vulgäre „Sommersprossen“ anzusprechende symmetrische Pigmentirung fällt jedoch weniger auf wie eine blos die linke Gesichtshälfte occupirende merkwürdige Hautveränderung, die sich aus vier disseminirten durch (abgesehen von der Pigmentirung) gesunde Haut getrennten Herden zusammensetzt. Zunächst fesselt den Blick eine lochförmige Vertiefung inmitten der linken Schläfe. Die steil abfallenden, nach der Stirnhaargrenze in weissliches Narbengewebe auslaufenden Ränder, in denen man die kleinen Grübchen geheilter Nadelstichcanäle wahrnimmt, zeigen, dass eine Operationswunde vorliegt. Während die Ränder von hellröthlicher Farbe sind, ist der Grund der Wunde, besonders in der Richtung gegen das Auge hin, mit gelblichem Eiter bedeckt, der sich, mit den Herzschlägen isochron, hebt und senkt. Nach vorsichtiger Entfernung des Eiters zeigt sich am ocularen Ende der Wunde eine grauglänzende Haut, die 
gleichfalls pulsirt. Es ist die durch die Operation freigelegte Dura mater.

Den zweiten Herd bildet ein nur wenig erhabener flacher, bandförmiger, derb infiltrirter Streifen unmittelbarvor dem linken Ohre, etwa rom oberen Rande der Obrmuschel bis einen $\mathrm{Cm}$. über den unteren Rand derselben hinab. Hier nimmt auch die in der Ohrhöhe $1 \mathrm{Cm}$. betragende Breite plötzlich um das Doppelte zu. Der Sireifen besteht aus bläulich rothem glänzenden, derben, über der Unterlage nicht verschieblichen Gewebe, gegen die Mitte ist der Grund geschwürig zerfallen und mit übelriechendem Eiter bedeckt, nach dessen Entfernung leicht blutende Granulationen erscheinen.

Als dritter Herd zeigt sich hinter und unter dem linken Ohre ein kirschgrosser prominenter, runder, mit der Unterlage verwachsener Knoten mit eiterbedeckter, drusiger, gelbrother Oberfläche.

2 Cin. von diesem entfernt befindet sich ein grösserer Knoten, fast zirkelrund, stark prominirend, von derselben Beschaffenheit, der nach unten, gegen die seitliche Halsgegend, in blauröthliches Narbengewebe ausläuft, in dessen Mitte eine kleine eitrige Ulceration sich zeigt. Das ist der vierte Herd.

Die lymphdrüsen vor und hinter dem Ohre, am Unterkieferwinkel, vor und hinter dem Sternomastoidens der linken Seite sind stark geschwellt, theilweise mit der Haut verwachsen.

Im Uebrigen ist die Gesichtshaut vollkommen normal zart, glatt und weich. Nirgends ein e Spur von Schuppung, Narbenbildung oder Atrophie.

Die Diagnose hatte zu entscheiden zwischen Lupus vulgaris, Mykosis fungoides, Sarkom und Carcinom. An Syphilis konnte bei der Abwesenheit jeder anderen Erscheinung und dem klinischen Aussehen der Geschwïlste ïberhaupt nicht gedacht werden. Der bandförmige Streifen vor dem Ohre sah Lupus vulgaris sehr ähnlich, dagegen sprach jedoch das überaus rasche Entstehen und Wachsthum innerhalb zweier Monate, auch die Unverschieblichkeit über der Unterlage. Die Knoten unter dem 
Ohre hatten das Aussehen von Lupus papillomatosus. Aber letzterer setzt doch lupöse Beschaffenheit oder wenigstens narbige Spuren einer solchen am Standort der Wucherungen roraus und nicht Herauswachsen derselben aus ganz gesunder Haut wie hier. In seltenen Fällen sieht man allerdings auch einen Lupus vulgaris in Tumorenform. Aber diese Tumoren sind weich, mit der Unterlage nicht verwachsen und bestehen seit früher Jugend. Gegen Mykosis fungoides sprach der Mangel eines ekzematösen Vorstadiums, die isolirte Beschränkung der Geschwülste auf das Gesicht und das Alter des Kranken, da meines Wissens Mykosis fung. vor dem 25. Lebensjahre bislang nicht beobachtet worden ist.

Die Unbeweglichkeit dieser derben Geschwülste, die Verwachsung mit der Unterlage, das gleiche Verhalten der Lymphdrüsen in Verbindung mit dem rapiden Entstehen und dem cachektischen Aussehen des Kranken mnsste sofort den Gedanken an einen malignen Tumor wachruten, der mit Rücksicht auf das klinische Verhalten nur Carcinom sein konnte. Die mikroskopische Untersuchung bestätigte diesen Verdacht. Sie ergab den Befund eines typischen Epithelialcarcinoms. ')

Obwohl nun eines der von Kaposi hervorgehobenen Hauptmerkmale, die trockene atrophische Haut, gänzlich fehlte, war es doch ausser Zweifel, dass hier ein Fall von Morbus Kaposi vorlag. Denn das Essentielle und Markante des Processes scheint mir doch in der Coincidenz von Epheliden, Teleangiektasien und multiplen Hautcarcinomen bei jugendlichon Individuen zu liegen. Mit Rücksicht auf das Fehlen der Xerodermie sehe ich mich aber veranlasst, der Etikette Kaposi's die Pick'sche Bezeichnung voranzustellen, da sie hier jedenfalls die treffendste ist.

Bei der Aufnahme des Kranken konnte von einer Behandlung des Grundleidens zunächst keine Rede sein. Wie schon in der Anamnese erwähnt, traten Anfälle von heftigem Kopfschmerz und Erbrechen auf, bei gleichzeitigem Bestehen einer stark

1) Eine Beschreibung der mikroskopischen Präparate unterlasse ich, da sie nur die bekannten histologischen Details wiederholen müsste. 
secernirenden, bis auf die Dura reichenden Kopfwunde die sicheren Anzeichen einer meningitischen Reizung. Dieselbe konnte man nicht lediglich dem Offenliegen der Dura zuschreiben. Es bestand offenbar eine Eiterretention. Liess man den Kopf zur linken Seite neigen, so zeigte sich am ocularen Ende der vorher abgetupften Wunde sofort ziemlich Eiter, der deutlich zwischen Dura und Schädel austrat. Der Kranke wurde nun sofort ins Bett beordert und angewiesen, die linke Kopfseite auf die Kissen aufzulegen. Im Verlauf einer Stunde ergoss sich über $1 / 4$ Liter Eiter aus der Wunde. Kopfschmerz und Erbrechen waren beseitigt.

Die Erklärung hierfür dürfte wohl sein: der Knabe hielt merkwürdiger Weise den Kopf stets nach der rechten, der Wunde entgegengesetzten Seite geneigt. Hierdurch sowie durch einen fest anliegenden Verband wurde der Eiter am Abfluss gehindert und bahnte sich einen Weg zwischen Dura und Schädel, einen künstlichen Epiduralraum schaffend, aus dem er bei Linkslage nach dem Gesetz der Schwere wieder ausfloss.

Die Wunde wurde nun täglich mit Borwasser ansgewaschen, $2 \%$ Salicylsalbe lose verbunden und der Knabe angehalten, den Kopf stets nach der linken Seite zu neigen. Alle 8 Tage wurde der Geschwürsgrtund leicht mit Lapis touchirt. Unter dieser Behandlung wurde die Secretion bald spärlich, aber es dauerte 6 Monate, bis sie ganz versiegt und die Wunde sich geschlossen hatte. Ein knöcherner Wiederersatz des operativ entfernten Stäckes fand nicht statt. Der Grund der geheilten Wunde fühlt sich noch jetzt hautartig weich an.

Nach Beseitigung der meningitischen Symptome wäre die operative Entfernung der Carcinome angezeigt gewesen. Ich musste mich gegen eine solche aussprechen. Die Exstirpation dieser 3 Carcinomherde in Verbindung mit der absolut nothwendigen Ausräumung und Entternung der wahrscheinlich gleichfalls carcinomatösen Drüsen stellte eine höchst gefährliche Operation vor. Es war wenig wahrscheinlich, durch sie alles Kraukhafte, insbesondere die tiefer gelegenen Drüsen zu entfernen. Es war ferner schwer anzunelmen, dass der äusserst heruntergekommene, anämische, kraftlose, zitternde Knabe eine so 
schwere Operation überstehen würde. Man musste sich endlich sagen - und dies ist der Haupteinwand - dass hier nicht ein solitäres Carcinom vorlag, dessen nothwendige Entfernung ausser Frage steht. sondern eine Carcinosis, eine Neigung zu multipler Carcinombildung. Waren doch der operativen Entfernung des erstens Knotens in kürzester 7eit drei weitere Knoten und hochgradige Drüsenschwellung gefolgt. Jeden Augenblick war das Erscheinen eines neuen Carcinomherdes zu erwarten. Der weitere Verlauf sollte mir Recht geben.

Unter diesen Umständen blieb Nichts übrig, als auf den Arsenik, das mächtigste Alterans der gesammten materia medica, zu recurriren. Man durfte hoffen, die Carcinosis günstig zu beeinflussen und dadurch das Leben des Kranken wenigstens zu verlängern. Am liebsten hätte ich subcutane Arsen-Injectionen nach Köbner vorgenommen, die rationellste und wirksamste Form der Arsen-Medication. Bei dem Zustande des Patienteu fürchtete ich aber den Schmerz der Procedur und das Entstehen von Abscessen. Ich verordnete daher die Pilulae asiaticae, à $3 \mathrm{Mg}$, liess mit einer täglich beginnen und stieg bis auf 3 . Bei dieser Dosis blieb ich, da grössere nach meiner Frfahrung keine ersichtlich bessere Wirkung zeigen.')

In den ersten zwei Monaten blieb der Zustand gleich. Aber nach Verbrauch von 200 Pillen trat innerbalb weniger Tage eine auffällige Veränderung ein. Die Knoten wurden flach, trockneten ein, fielen endlich ab und hinterliessen eine bräunliche Kruste, die unter Zurücklassung einer blauröthlich verfärbten, fast glatten, allerdings infiltrirten Haut rasch verschwand. Ende 1888 war der Zustand des Knaben ein glänzender zu nennen. Die Stirnwunde war geheilt, die Carcinomknoten verschwunden, der Allgemeinzustand durch Hebung des Appetits vortrefflich geworden. Auch die Drüsenschwellung war bedeutend zurïckgegangen.

5) Es ist in letzter Zeit Mode geworden, Arsenik-Pillen keratinirt zu verordnen. Wie der altübliche Zusatz von Piper beweist, beabsichtigt man, gerade durch den Magensaft die an sich unlösliche und deshalb vielfach proscribirte arsenige Säure löslich zu machen. Die Keratinirung, welche jede Einwirkung des Magensaftes ausschliesst, muss daher als eine irrationelle Darreichungsform bezeichnet werden. 
So blieb es bis Anfung Mai 1890. Um diese Zeit bildete sich am Unterkiefer, genau in der Mitte zwischen Kinn und Ohr, eine ovale in der Haut gelegene hellröthliche Prominenz, die unter starken Schmerzen langsam wuchs, fluctuirend wurde und ziemlich Eiter entleerte. Bald verwandelte sich der scheinbare Abscess in einen gelbrothen, drüsigen stark prominenten Knoten. Gleichzeitig wuchsen in wenig Tagen auch die scheinbar geheilten Carcinomknoten vor und unter dem Ohre wieder empor.

Der Arsenik bewährte sich wiederum. Bereits Ende Juli war an Stelle der Knoten die Hant vur noch blcuroth verfürbt. Da begann im August der Knabe über Schmerzen in der Gegend des Promontorium zu klagen. Die Haut daselbst war intact, aber stark vorgewölbt durch einen derbteigigen Tumor, der von der Wirbelsäule ausging. Unter Zunahme der Schmerzen röthete sich die Haut an den beiden Enden des Tumors. Es entleerte sich eine grosse Eitermenge, worauf sich an den Entleerungsstellen zwei tiefe Geschwüre bildeten, in deren Grund papilläre Wucherungen erschienen. Unter Fortgebrauch des Arseniks und Verband mit $2 \%$ Salicylsalbe waren die Geschwüre bis October zugeheilt. Der Tumor selbst blieb unverändert. An seiner sarco-carcinösen Natur ist nicht zu zweifeln.

Der Zustand jetzt, Ende Februar 1891, ist folgender: Die Schädelwunde geheilt; die Stelle vor dem Ohre überhäutet, aber roth und infiltrirt. Die Knoten unter dem Ohre verschwunden, an ihrer Stelle die Haut blauroth schuppend, derb infiltrirt und bretthart mit der Unterlage verwachsen. Der Knoten in der Mitte des Unterkiefers hat sich in eine rothe, derbe iiberbüutete keloidartige Prominenz verwandelt. Die Lymphdrüsen der linken Seite sind stark geschwellt und vielfach mit der Haut verwachsen. In der Gegend des Promontorium ein $3 \mathrm{Cm}$. über die Umgebung hervorragender, von der rechten zur linken anteren Lumbalregion sich erstreckender Tumor, mit normaler, nur an den beiden Endpunkten röthlich schuppender Haut überzogen. Alle diese Geschwülste auf Druck nicht schmerzhaft, wohl aber zuweilen spontan. Das Allgemeinbefinden ist gut. Ueber die fatale Prognose und den schliesslichen Ausgang kann trotzdem kein $\mathrm{Zweifel} \mathrm{sein.}$ 
Von Interesse duirfte sein, dass der Kranke bis jetzt an 3000 asiatische Pillen à 0,003 verbraucht und vorzüglich vertragen hat. Nur einigemal traten Diarrhoe und Leibschmerzen auf, wurden aber durch Heruntergehen der Dosis rasch bes eitigt. Seit Anfang März nimmt er nur noch 2 Pillen täglich. Ich werde auch diese Dosis noch reduciren, aber eine Arsenikpille gedenke ich fortwährend zu geben. Ich beabsichtige mit einem Wort, den Knaben zu einem Arsenikesser zu machen, da ich keine andere Möglichkeit sehe, sein Leben zu verlängern.

Ich möchte den Fall nicht abschliessen, ohne über die Aetiologie gesprochen zu haben. Dass an der Pigmentirung starke Besonnung nicht Schuld ist, geht aus der Anamnese hervor. Es scheint mir überbaupt unwahrscheinlich, dass circumscripte Pigmentirungen unter dem Einfluss der Sonnenstrahlen zu Stande kommen, da man sie doch an Stellen sieht, wo für gewöhnlich die Sonne nicht hindringt, nämlich an den Genitalien. Durch häufige Besonnung sieht man nur diffuse, im Winter cessante causa stark abblassende Pigmentirung der entblösst getragenen Körpertheile entstehen, wovon man sich bei unseren Landleuten täglich überzeugen kann. Für die ephelidenartigen Bildungen scheint mir die Annahme einer hereditären, seltener erworbenen nervösen Pigmentverschiebung am plausibelsten.

Was die Carcinosis betrifft, so liegt es am nächsten, das in der Kindheit bestandene und nach der Heilung wieder künstlich herrorgerufene Ekzem mit der gegenwärtigen Erkrankung in Zusammenhang zu bringen. Es befand sich an derselben Stelle wie der erstentstandene Carcinomknoten. Man darf *ielleicht annehmen, dass die Mikroorganismen des Carcinoms damals zufällig auf die epidermisberaubten Stellen gerathen, mit eingeheilt und nach langer Incubation die gegenwärtige Erkrankung verursacht haben. Man weiss allerdings noch Nichts von diesen Carcinomerregern. Aber dass es solche geben muss, und dass dieselben ein Incubationsstadium besitzen, welche dasjenige aller bekannten Infectionskrankheiten vieltausendfach übertrifft, geht aus allen klinischen Thatsachen herror. Hier ist nicht der Ort, näher darauf einzugehen.

Auch in den übrigen bisher beobachteten Fällen der Kaposi'schen Krankheit nehme ich keinen inneren Zusammenhang 
zwischen den vorgefundenen Sarkomen resp. Carcinomen und der „buntscheckigen" Haut an, sondern betrachte die Tumoren nur als die Folgen der Infection einer hochgradig schwachen und vulnerablen Haut.

Die beigegebene Tafel XII. veranschaulicht den Zustand im Júli 1888. Nur den später hinzugekommenen Knoten am Unterkiefer habe ich im Mai 1889 nachträglich eingezeichnet. 


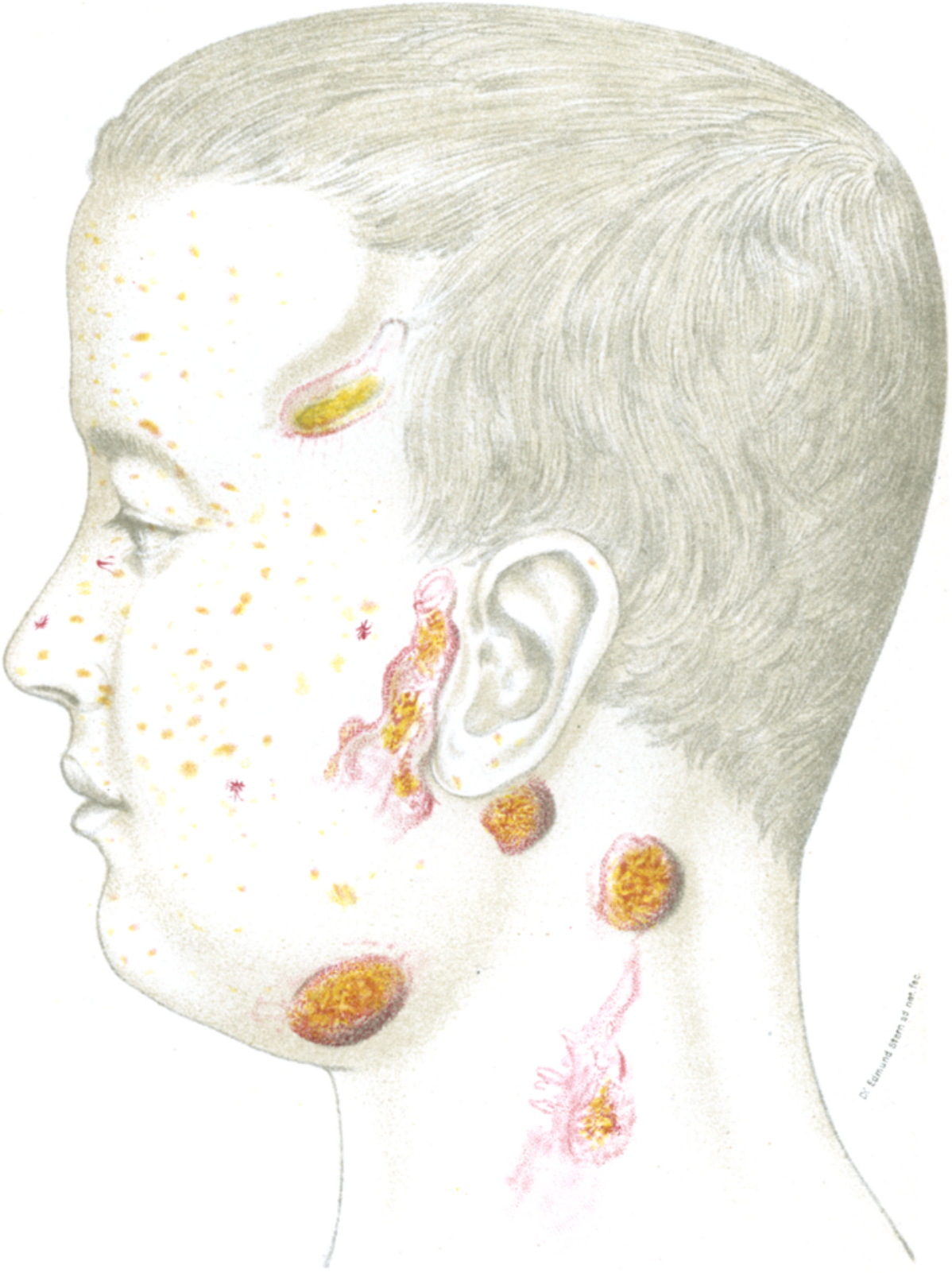

